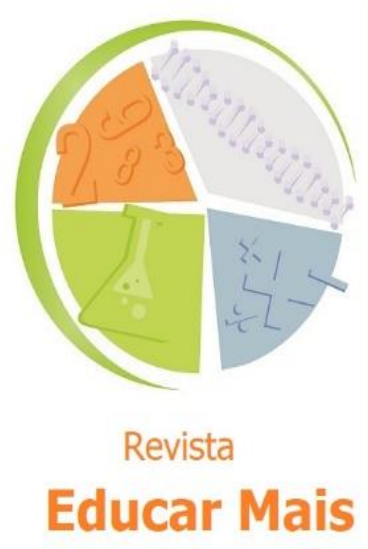

\title{
As contribuições de um agente conversacional no ensino e aprendizagem da Física: uma revisão de literatura
}

\author{
The conversational agents' contributions in teaching and learning \\ Physics: a literature review
}

\section{Las contribuciones de los agentes conversacionales en la enseñanza y el aprendizaje de la Física: revisión de la literatura}

Kátia Martins Soares ${ }^{1}$; Liane Margarida Rockenbach Taroucoㄹ; Patrícia Fernanda da Silva ${ }^{3}$

\begin{abstract}
RESUMO
O artigo aborda as contribuições do agente conversacional/chatbot/tutor virtual no ensino e aprendizagem dos conteúdos da Física. O chatbot é um programa que simula um diálogo entre o homem e um robô em linguagem natural. A pesquisa bibliográfica contempla estudos internacionais e nacionais, mediado pela busca das palavras-chave: (chatbot, education, high school, physics); com recorte temporal de 2016-2020, utilizou-se 08 artigos que atendiam aos critérios de inclusão. Os benefícios para aprendizagem compõem-se: estabelecimento de uma metodologia ativa de ensino, proposição de mais um "espaço virtual" para receber feedback; capacidade de simular experimentos, proporcionando uma alternativa à escassez de laboratórios de Ciências. $O$ agente conversacional, comparado a outros métodos pedagógicos, demonstra-se superior à leitura de um livro sobre os mesmos conteúdos, em tempos iguais. A evolução na aprendizagem foi equivalente em pesquisas relacionando o tutor virtual e tutores humanos, prevalecendo os tutores humanos em situações de esclarecimento de dúvidas.
\end{abstract}

Palavras-chave: Chatbot; Física; Educação; Ensino Médio; Aprendizagem.

\begin{abstract}
The article addresses the contributions of the conversational agent/chatbot/virtual tutor in teaching and learning the contents of Physics. The chatbot is a program that simulates a dialogue between man and a robot in natural language. The bibliographic research includes international and national studies, mediated by the search for keywords: (chatbot, education, high school, physics); with a 2016-2020 time frame, 08 articles were used that contemplate the inclusion criteria. The benefits for learning are: establishment of an active teaching methodology, proposing yet another "virtual space" to receive feedback; ability to simulate experiments, providing an alternative to the scarcity of science laboratories. The conversational agent, compared to other pedagogical methods, proves to be superior to reading a book on the same contents, at equal times. The
\end{abstract}

\footnotetext{
${ }^{1}$ Doutoranda no Programa de Pós-Graduação em Informática na Educação (PPGIE) da Universidade Federal do Rio Grande do Sul (UFRGS) e Professora dos anos finais no Ensino Fundamental no município de Canoas/RS - Brasil. E-mail: prof.katiasoares@gmail.com

${ }^{2}$ Licenciada em Física e Doutora em Engenharia Elétrica/Sistema Digitais. Professora titular da Universidade Federal do Rio Grande do Sul (UFRGS) e atua junto ao Programa de Pós-Graduação em Informática na Educação, Porto Alegre/RS - Brasil. E-mail: liane@penta.ufrgs.br

${ }^{3}$ Graduada em Ciências Exatas com habilitação integrada em Matemática, Física e Química, Mestre em Ensino de Ciências Exatas, Doutora em Informática na Educação e Professora colaboradora do Programa de PósGraduação em Informática na Educação (PPGIE) da Universidade Federal do Rio Grande do Sul (UFRGS), Porto Alegre/RS - Brasil. E-mail: patriciasilva@mail.com
} 
evolution in learning was equivalent in research relating the virtual tutor and human tutors, with human tutors prevailing in situations of clarification of doubts.

Keywords: Chatbot; Physics; Education; High school; Learning.

\section{RESUMEN}

El artículo aborda las aportaciones del agente conversacional/chatbot/tutor virtual en la enseñanza y aprendizaje de los contenidos de Física. El chatbot es un programa que simula un diálogo entre un hombre y un robot en lenguaje natural. La investigación bibliográfica incluye estudios internacionales y nacionales, mediados por la búsqueda de palabras clave: (chatbot, educación, bachillerato, física); con un marco temporal 2016-2020, se utilizaron 08 artículos que contemplan los criterios de inclusión. Los beneficios para el aprendizaje son: establecimiento de una metodología de enseñanza activa, proponiendo otro "espacio virtual" para recibir retroalimentación; capacidad para simular experimentos, proporcionando una alternativa a la escasez de laboratorios de ciencias. El agente conversacional, comparado con otros métodos pedagógicos, resulta superior a leer un libro sobre los mismos contenidos, en momentos iguales. La evolución en el aprendizaje fue equivalente en las investigaciones que relacionan al tutor virtual y los tutores humanos, predominando los tutores humanos en situaciones de aclaración de dudas.

Palabras clave: Chatbot; Física; Educación; Escuela secundaria; Aprendizajes.

\section{INTRODUÇÃO}

O estudo sobre a aprendizagem é decorrente de pesquisas extensas, as quais subsidiam a forma como o ser humano aprende. Ela é complexa, envolve fatores internos do ser humano e externos em relação ao meio e objetos. Frente às conquistas tecnológicas dos últimos anos, vem expandindo-se o interesse científico na forma como a aprendizagem pode ser mediada pelas ferramentas tecnológicas e seus benefícios para educação. Neste aspecto, o objetivo deste artigo é levantar as contribuições de um agente conversacional, investigando a sua potencialidade de interação, colaboração e mediação no ensino e aprendizagem de Física. Assim como, refletir sobre a teoria que subjaz esta aprendizagem ou a metodologia de ensino.

A teoria Sócio Interacionista de Vygotsky (1989) relaciona o aprendizado decorrente das interações sociais e tem sido utilizada em algumas abordagens envolvendo o uso da tecnologia (ARRUDA e CASTRO 2018; TAROUCO, 2018). Conforme proposto por Vygotsky na publicação Mind in Society (1930) há um conceito de Zona de Desenvolvimento Proximal, definido como a distância entre o nível de desenvolvimento real, determinado pela solução independente de problemas, e o nível de desenvolvimento potencial ${ }^{4}$, conforme determinado pela solução de problemas sob a orientação de um adulto ou em colaboração com colegas mais capazes. No presente trabalho, o agente conversacional é proposto como sendo capaz de assumir o papel de um companheiro virtual mais capacitado, que pode alavancar o desenvolvimento do estudante.

Os agentes conversacionais ou chatbots são softwares programados com inteligência artificial que simulam a fala humana e interagem em diversos setores; tiveram seus primórdios em empresas para atender clientes, prestar informações sobre serviços, vender produtos e entregar conteúdo.

\footnotetext{
${ }^{4}$ A expressão desenvolvimento potencial foi usada na versão em Inglês do livro Mind in Society, publicado em 1978 pela Harvard University Press. Na versão para o Português a obra, traduzida sob a coordenação da Universidade de São Paulo, em 1989, usou a expressão desenvolvimento proximal.
} 
Atualmente, tornou-se mais frequentemente no ensino superior (LEONHARDT, 2003; TAROUCO, SILVEIRA e KRASSMANN 2018).

O uso das ferramentas, aplicativos e softwares disponíveis para mediar o ensino e a aprendizagem da Física justifica-se por ser uma disciplina com necessidade de desenvolvimento das habilidades de pensamento superior, como destacado por Silitonga (2020). Além disso, nos últimos anos, apresentou mudanças bruscas no currículo. Dentre estas, a redução da carga horária semanal; necessidade de fomentar o uso da TIC (Tecnologia da Informação e Comunicação) para que as aulas tornem-se mais atrativas. Autores sugerem uma transformação na forma de ensinar, passando de uma abordagem tradicional para uma metodologia ativa de ensino, permeada pela tecnologia (MOREIRA, 2018, ROSA, 2012).

Segundo Teixeira (2019) destacam-se três relevantes aspectos ligados ao ensino e à aprendizagem no ensino de Ciências: o livro didático, a experimentação, e a inserção da abordagem histórica e historiografia (estudo e descrição da história). Neste aspecto, a subutilização dos recursos tecnológicos, não contribui para o avanço histórico e para o preparo dos alunos para atuarem no presente; dificultando a formação dos cidadãos para a sociedade científica e tecnológica.

Dados levantados pelo Instituto de Estudo e Pesquisa (INEP, 2018) referem-se à escassez da infraestrutura de laboratórios de Ciências. Dentre as escolas pesquisas: 95,1\% das escolas de Ensino Médio (EM) possuem acesso à internet, o laboratório de ciências em apenas 44,1\% delas; somente $38,8 \%$ das escolas de EM da rede pública, e 57,2\% na rede privada possuem laboratório de Ciências; $87,5 \%$ de todas as escolas do EM possuem biblioteca; 78,1\% laboratório de informática (INEP, 2018).

Destaca-se que a ausência de atividade de experimentação afeta a aprendizagem, pois sem as aulas práticas, o estudante não tem como observar, interagir, refletir. Heck et al. (2016) mencionam que a forma como são apresentados os conceitos, leis e fórmulas na Física não promovem aprendizado e não se aproximam da vivência dos alunos.

Torna-se relevante buscarem-se alternativas para além do uso do livro didático e para a falta de laboratórios de ciências. Pesquisas recentes demonstram que os laboratórios de informática podem ser uma alternativa, desde que acompanhem a evolução das novas ferramentas disponíveis para a educação (HERPICH, 2019). Ademais, é crescente o uso de dispositivos móveis e das mídias sociais para mediar, apresentar, discutir, realizar testes e experimentos, entre outras funções, que tornam as aulas mais motivadoras e envolventes (DANTAS et al. 2019).

O artigo está dividido a partir desta introdução, da seguinte forma. Na seção 2 apresenta-se a metodologia empregada, caracterizada por uma revisão sistemática qualitativa, cujos estudos primários foram integrados de forma sintetizada, contemplando os descritores de busca, os critérios de inclusão e exclusão, bem como os artigos utilizados após análise criteriosa. Na seção 3, a revisão teórica apresentando o entrelaçamento entre a Física, teoria de aprendizagem e a tecnologia subdividindo-se em 3.1 Aprendizagem: Teoria Interacionista de Vygotsky; 3.2 metodologias ativas de ensino; 3.3 ensino de Física; 3.4 agente conversacional apoiando a Física, apresentando os trabalhos pesquisados, os conteúdos abordados, as ferramentas para programação. A seção 4 finaliza com as considerações finais do artigo e trabalhos futuros. 


\section{METODOLOGIA ABORDADA}

O estudo de revisão sistemática segue as diretrizes previamente descritas por autores de referência da área. De acordo com (KLOCK, 2018) as características que envolvem a Revisão Sistemática de Literatura (RSL) em relação ao objetivo geral abarcam a profundidade, envolvendo uma escolha específica na questão da pesquisa, cujo escopo envolve uma pequena quantidade de estudos, e a revisão envolve a análise de qualitativa.

As revisões sistemáticas são uma forma de avaliar e interpretar toda a pesquisa relevante sobre um interesse específico. Neste sentido, as revisões analisam um conjunto menor de estudos, pois tem objetivos peculiares e focam em aprofundar os conhecimentos já existentes em um determinado tema, como a comparação entre a eficácia de diferentes métodos (KITCHENHAM, 2009).

O critério de inclusão contempla pesquisas aplicadas envolvendo o uso de chatbot na disciplina de Física no Ensino Médio, bem como no nível superior em Física. Consequentemente foram excluídas outras disciplinas. Inicialmente pesquisou-se a base de dados da Scopus ${ }^{5}$ considerada o maior banco de dados de resumos e citações de artigos para jornais e revistas acadêmicos. Usou-se ["chatbot" and "high school" and "physics"] retornando 10 resultados, dos quais não abordavam o ensino na disciplina de Física e não foram elencados.

Na plataforma do Google Scholar ${ }^{6}$ com os mesmos descritores: ["chatbot" and "high school" and "physics"] retornaram 407 resultados. O critério de inclusão para seleção do artigo exigia que estivesse contemplado no título ou nas palavras chaves a disciplina de física ou algum conteúdo da mesma. (Outro critério foi em relação ao período de buscas, considerando de 2016 a 2020).

No entanto, algumas pesquisas anteriores ao período pré-definido, apresentam maior especificidade com o tema, como no caso de Graesser et al. (2004) e foram acrescentadas por contemplarem pesquisas recentes do autor. A pesquisa de Tarouco et al. (2018) sobre aprendizagem colaborativa com entidades virtuais foi incluída, pois evidencia a contribuição sobre a aprendizagem mediada pelos agentes virtuais.

No portal de periódicos da CAPES ${ }^{7}$ retornaram 22 resultados apenas mudando-se os descritores de chatbot and high school and physics para os descritores: chatterbot/chatbot and education, dos quais não foram utilizados na pesquisa.

A pesquisa está composta por 8 (oito) artigos que cumpriram os critérios previstos. Para a análise qualitativa averiguou-se qual nível educacional foi abordado, quais os conteúdos de Física foram apoiados com a tecnologia do chatbot, e qual teoria de aprendizagem ou metodologia permeia as ações. A Tabela 1 ilustra os artigos que compõe a presente pesquisa:

\footnotetext{
${ }^{5}$ https://www.scopus.com/results/results.

${ }^{6}$ https://scholar.google.com.br/scholar

${ }^{7}$ http://www.periodicos.capes.gov.br
} 
Tabela 1- Pesquisas relacionadas

\begin{tabular}{|c|c|c|c|c|}
\hline Título & Autor & $\begin{array}{c}\text { Nível } \\
\text { educacional/ } \\
\text { Conteúdo da } \\
\text { Física }\end{array}$ & $\begin{array}{c}\text { Recurso } \\
\text { Tecnológico }\end{array}$ & $\begin{array}{c}\text { Forma de mediação } \\
\text { do chatbot/ Teoria } \\
\text { de Aprendizagem, } \\
\text { Metodologia }\end{array}$ \\
\hline $\begin{array}{l}\text { 1.Learning Principles } \\
\text { of Electricity Through } \\
\text { Experiencing in Virtual } \\
\text { Worlds }\end{array}$ & $\begin{array}{l}\text { Herpich F., } \quad \text { Filho } \\
\text { T.A.R., Tibola L.R., } \\
\text { Ferreira V.A., Tarouco } \\
\text { L.M.R. (2017) }\end{array}$ & $\begin{array}{l}\text { Ensino Médio } \\
\text { Eletricidade }\end{array}$ & $\begin{array}{l}\text {-AIML } \\
\text {-Mundo } \\
\text { Virtual Imersivo }\end{array}$ & $\begin{array}{l}\text { Aprendizagem } \\
\text { Experiencial de Kolb }\end{array}$ \\
\hline $\begin{array}{l}\text { 2.Collaborative } \\
\text { Learning with Virtual } \\
\text { Entities. }\end{array}$ & $\begin{array}{l}\text { Tarouco, L. M. } \text { R. } \\
\text { Silveira, } \\
\text { C. Krassmann, A. } \\
(2018)\end{array}$ & $\begin{array}{l}\text { EAD } \\
\text { Ensino Superior }\end{array}$ & $\begin{array}{l}\text { AIML } \\
\text { ALICE }\end{array}$ & $\begin{array}{l}\text { Teoria Sócio } \\
\text { Interacionista de } \\
\text { Vygotsky. Metodologia } \\
\text { ativa com aprendizagem } \\
\text { baseada em problemas } \\
\text { (PBL) }\end{array}$ \\
\hline $\begin{array}{l}\text { 3.A computer-based } \\
\text { tutor for learning } \\
\text { energy and power. }\end{array}$ & $\begin{array}{l}\text { Aravind, V. } \quad \text { R., } \\
\text { Mcconnell, } \\
\text { (2018). }\end{array}$ & $\begin{array}{l}\text { Ensino Médio } \\
\text { Energia } \\
\text { potência }\end{array}$ & $\begin{array}{l}\text { Plataforma } \\
\text { TutorShop }\end{array}$ & Metodologia ativa PBL \\
\hline $\begin{array}{lr}\text { 4.AstroBot: } & \text { Um } \\
\text { chatbot } & \text { com } \\
\text { inteligência artificial } \\
\text { pararaxiliar } & \text { no } \\
\text { processo de ensino e } \\
\text { aprendizagem r de } \\
\text { Física. }\end{array}$ & $\begin{array}{l}\text { Dantas, A. C. Torres, } \\
\text { H. Faria, I. P. Araújo } \\
\text { W. Guimarães } \\
\text { G.Machado G.Almeida. } \\
\text { A. V. Nascimento. M. } \\
\text { Z. (2019). }\end{array}$ & $\begin{array}{l}\text { Ensino Médio } \\
\text {-velocidade } \\
\text {-cinemática } \\
\text {-vetores } \\
\text {-movimento } \\
\text { uniformemente } \\
\text { variado } \\
\text {-trajetória }\end{array}$ & $\begin{array}{l}\text { AIML } \\
\text { PYTHON } \\
\text { Dialogflow } \\
\text { Web } \\
\text { responsive: } \\
\text { acesso } \\
\text { dispositivo } \\
\text { móvel }\end{array}$ & Teorias construtivistas \\
\hline $\begin{array}{l}\text { 5.AutoTutor: A tutor } \\
\text { with dialogue in } \\
\text { natural language. }\end{array}$ & $\begin{array}{l}\text { GRAESSER, A.C., LU, } \\
\text { S., JACKSON, G.T. ET } \\
\text { AL. (2004) }\end{array}$ & $\begin{array}{l}\text { Ensino Médio } \\
\text { Física qualitativa } \\
\text { newtoniana }\end{array}$ & $\begin{array}{l}\text { Java }^{8} \\
\text { Arquitetura } \\
\text { Modular }\end{array}$ & $\begin{array}{l}\text { Teorias construtivistas } \\
\text { Estratégias de resolução } \\
\text { de problemas (PBL) }\end{array}$ \\
\hline $\begin{array}{lr}\text { 6.Learning } & \text { newtonian } \\
\text { physics ren with } \\
\text { conversational agents } \\
\text { and inderactive } \\
\text { simulations. }\end{array}$ & $\begin{array}{l}\text { Graesser, A. C., } \\
\text { Franceschetti, } \\
\text { Gholson, B., \& Craig, S } \\
(2018)\end{array}$ & $\begin{array}{l}\text { Ensino Médio e } \\
\text { Superior } \\
\text { Física qualitativa } \\
\text { newtoniana }\end{array}$ & $\begin{array}{l}\text { Java } \\
\text { Arquitetura } \\
\text { Modular }\end{array}$ & $\begin{array}{l}\text { Teorias construtivistas } \\
\text { Estratégias de resolução } \\
\text { de problemas (PBL) }\end{array}$ \\
\hline $\begin{array}{l}\text { 7.Development Of The } \\
\text { Chatbot Einstein } \\
\text { Application as a Virtual } \\
\text { Teacher of Physical } \\
\text { Learning in The House } \\
\text { Using Android Based } \\
\text { Google Dialogflow Api }\end{array}$ & $\begin{array}{l}\text { ANWARULLO, T. P. } \\
\text { AGUSTIA, R. D. }\end{array}$ & $\begin{array}{l}\text { Chatbot Einstein } \\
\text { Ensino Médio } \\
\text { Conhecimento } \\
\text { grandezas } \\
\text { (energia, massa, } \\
\text { tempo) }\end{array}$ & $\begin{array}{l}\text { AIML } \\
\text { Dialogflow } \\
\text { API para } \\
\text { Android }\end{array}$ & $\begin{array}{l}\text { Metodologia } \\
\text { através PBL }\end{array}$ \\
\hline $\begin{array}{l}\text { 8.ELEKTRA: } \\
\text { Chatterbot } \\
\text { em } \quad \text { um } \\
\text { Educacional }\end{array}$ & $\begin{array}{l}\text { Leonhardt, } \quad \text { M.D. } \\
\text { Castro, D. Dutra.R. L. } \\
\text { S. Tarouco, L. M. R. } \\
(2003)\end{array}$ & $\begin{array}{l}\text { ELEKTRA } \\
\text { Ensino Superior }\end{array}$ & $\begin{array}{l}\text { ALICE } \\
\text { AIML }\end{array}$ & Teorias construtivistas \\
\hline
\end{tabular}

Fonte: Elaborado pelos autores

\footnotetext{
8 Java é uma linguagem de programação orientada a objetos desenvolvida na década de 90 por uma equipe de programadores chefiada por James Gosling, na empresa Sun Microsystems. Em 2008 o Java foi adquirido pela empresa Oracle Corporation.
} 


\section{REVISÃO TEÓRICA}

O referencial teórico apresenta conceitos inerentes à Física enquanto campo de conhecimento; Teoria Sócio Interacionista de Vygotsky, Metodologias Ativas, na qual se compõe de diferentes técnicas de ensino: o ensino híbrido, construção de mapas conceituais, sala de aula invertida, aprendizagem baseada em problema (Based Problem Learning-PBL). Além disso, é composta sobre a tecnologia do agente conversacional e as contribuições na Física.

\subsection{Aprendizagem: Teoria Interacionista de Vygotsky}

De acordo com a teoria de Vygotsky o desenvolvimento cognitivo acontece com a transformação das relações sociais em funções mentais. No ser humano o desenvolvimento é mediado por instrumentos e signos produzidos no seu meio social, cultural e histórico (VYGOTSKY, 1989).

Com a socialização acontecem os processos mentais superiores, sendo o pensamento, a linguagem e a expressão da vontade originados nos processos sociais. Para Vygotsky (1989) a ação social do ser humano é mediada por instrumentos e signos que influenciam para que as condições das funções psicológicas se desenvolvam. Um exemplo é a fala, que demonstra os atos de compreensão e interpretação do meio que o cerca. Todos os signos não verbais, embora não possam ser substituídos por palavras, inserem-se no discurso, apoiam-se nas palavras e são acompanhados por elas: "a palavra não é uma coisa que o indivíduo possui, mas é mediação, elemento móvel e mutável na dinâmica das interações verbais" (MOREIRA, 2016, p.19).

Neste sentido, para compreensão da obra de Vygotsky, apresentam-se outras variáveis do desenvolvimento, cujos estudos instituem as acepções de: mediação, instrumentos e signos, interação social, significados, fala, Zona de Desenvolvimento Proximal (ZDP) os quais são descritos, brevemente. Nos seus estudos com primatas, Vygotsky (1989) inferiu que existia uma inteligência prática em ação, ao considerar que aqueles animais conseguem atingir um nível de resolução de problemas ao utilizarem instrumentos ao seu redor. A partir destes estudos, o autor formulou alguns conceitos: linguagem e pensamento, mediação semiótica, internalização, zonas de desenvolvimento.

Neste sentido, a teoria aponta que a criança nasce com funções psicológicas elementares e que com o aprendizado da cultura e as experiências adquiridas, essas funções tornam-se funções psicológicas superiores, demonstradas pelo comportamento consciente, a ação proposital, capacidade de planejamento e pensamento abstrato.

A linguagem e o pensamento foram elucidados comparando as expressões faciais dos primatas, tais como os urros e gritos na comunicação. Quando analisou crianças, ele notou que elas passam por um processo semelhante. Mas por volta dos dois anos de idade a linguagem pré-intelectual e pensamento pré-verbal evoluíam frente à necessidade de resolução de problemas, dando origem à linguagem intelectual e ao pensamento verbal.

O autor contribui para entendermos a capacidade humana de unir a linguagem ao pensamento para organizar a realidade. O pensamento deixa de ser biológico, tal como nos primatas, para se tornar histórico-social, diferenciando o homem dos outros animais (VYGOTSKY, 1998).

A partir do momento que acontece a fala, o vocabulário torna-se rico, a criança tende a questionar o que lhe intriga, sua linguagem e seu pensamento passam a ser internalizados. Isso ocorre em três momentos: fala social (ou exterior): a comunicação engloba elementos do entorno e tem como 
finalidade apenas a comunicação com adultos; fala egocêntrica: a prioridade não é ser ouvida pelo adulto, mas ela ainda não saber expressar sua fala apenas para si; fala interior: quando atinge a potencialidade da reflexão.

Na teoria de aprendizagem de Vygotsky, a mediação é um conceito central e representa a intervenção de um elemento intermediário numa relação. Os mediadores podem ser instrumentos ou signos. Caracteriza como instrumentos os objetos criados pelo homem para auxiliar nos seus propósitos, os signos são fatores psicológicos que auxiliam nos processos internos.

Nas palavras de Moreira (2016, p.20): "o instrumento é algo que pode ser usado para fazer alguma coisa, o signo é algo que significa alguma coisa". A linguagem e a matemática constituem-se em elementos articulados de signos, os quais devem ser empregados na atividade humana para o seu desenvolvimento cognitivo. $O$ uso de signos linguísticos (palavras) permite que o indivíduo se afaste cada vez mais de um contexto concreto e expresse o ocorre no nível intrapessoal.

Para Vygotsky o ambiente desempenha um papel fundamental no desenvolvimento intelectual da criança. No processo de internalização, o desenvolvimento ocorre de fora para dentro, de forma que a absorção do conhecimento advém do contexto, decorrente do processo de socialização, já que, quando se relaciona com os pares, a criança reconstrói as formas culturais, o pensamento, as significações e os usos das palavras.

Conforme anteriormente destacado, Vygotsky categorizou o processo de aprendizagem em dois níveis, que denominou de Zonas de Desenvolvimento. Zona de Desenvolvimento Real: refere-se às etapas já alcançadas pela criança e que permitem que ela solucione problemas de forma independente. Zona de Desenvolvimento Proximal: é a distância entre o que o aprendiz pode fazer de forma independente e o que ele é capaz de fazer com a ajuda de um parceiro mais capaz. Ou seja, é o caminho a ser percorrido até o amadurecimento e a consolidação de funções (VYGOTSKY, 1989). A compreensão do conceito da zona de desenvolvimento proximal torna-se importante para planejamento educacional, postulando como o momento adequado para elaborar estratégias pedagógicas para que $\mathrm{o}$ aluno possa evoluir no aprendizado.

A interação social é o meio fundamental para a transmissão das interações interpessoais para intrapessoal e além de acontecer entre os participantes do processo de aprendizagem colaborativa (estudantes, tutores, professor e outras pessoas envolvidas) pode também acontecer entre os estudantes e entidades virtuais como agentes conversacionais.

As contribuições acerca da Teoria de Vygotsky permitem o entendimento de que as tecnologias digitais; neste cenário o agente conversacional, uma tecnologia programada com Inteligência Artificial funciona como instrumentos tecnológicos, simbólicos e culturais de ensino e aprendizagem (ARRUDA e CASTRO, 2018; TAROUCO, 2018, TAROUCO et al. 2020).

Conforme Arruda e Castro (2018) no seu estudo, revelaram-se singularidades e características dos processos de ensino e aprendizagem, mediados pelas tecnologias digitais, tentando compreender como ocorre a aprendizagem. Com uma metodologia diversificada e com uso de recursos tecnológicos distintos, que posteriormente foram analisados em grupo, encontrou-se um contexto fomentador de ZDP.

Dentre os parâmetros identificadores da ZDP descritos pelas pesquisadoras consideram-se: "espaços de diálogos entre o aprendente e pares e/ou alguém mais experiente; compreensão e/ou constituição 
de um novo conceito; compreensão conjunta; e mudanças conceituais e mediação, ou seja, através das ações intermediadas entre sujeito e objeto" (ARRUDA e CASTRO, 2018, p. 89).

Desta forma, demanda-se uma modificação na educação, passando-se para um processo de metodologia ativa de ensino e aprendizagem, cuja característica principal é a inserção do aluno/estudante como agente responsável pela sua aprendizagem, comprometendo-se com seu aprendizado. Esta metodologia é apresentada na próxima seção.

\subsection{Metodologias Ativas de Ensino}

O modelo de aula expositiva, em que o professor apresenta determinados conteúdos tem dado espaço às novas formas de ensinar, em que o professor passa a ser um mediador na aprendizagem do aluno, e este se torna responsável pelo aprendizado. Esta mudança de paradigma é denominada de metodologia ativa. Conforme Mota et al. (2018) a metodologia ativa abrange métodos e abordagens que estimulam a interação aluno-professor, aluno-aluno e aluno-materiais/recursos didáticos e envolvem, geralmente, a aprendizagem em um ambiente colaborativo.

Resumidamente são apresentadas abordagens que caracterizam as Metodologias Ativas: 0 ensino híbrido é uma modalidade de ensino que agrega a prática em sala de aula às aulas online através do uso da tecnologia para tarefas e avaliações à distância. O ensino híbrido já tem uma trajetória no ensino superior, alastrando-se para outros níveis educacionais. As modalidades de ensino on-line e presencial se complementam.

Os mapas conceituais são descritos como ferramentas gráficas que organizam visualmente conceitos e ideias, ajudando a explicar as conexões entre diferentes conceitos. A teoria dos mapas conceituais foi desenvolvida por Joseph Novak na década de 1970. O autor trabalhava com numerosos dados de entrevistas clínicas piagetianas e precisava de um instrumento para organizá-los; fundamentou-se na teoria da aprendizagem significativa de David Ausubel. Para Ausubel os novos conhecimentos se fixam quando estão relacionados com aquilo que já se conhece, ou seja, quando as ideias já existentes são modificadas a partir de novas ideias, estabelecendo conexões (MOREIRA, 2012).

Na sala de aula invertida o aluno tem acesso aos conteúdos online antes da aula presencial. Dessa forma, ele chega à sala de aula munido de informações adequadas, para debater o tema com os colegas e professores. Aprendizagem baseada em problema (Based Problem Learning-PBL): proposição de contextos, ou exercícios em que os alunos buscam a descoberta de soluções para este desafio. O objetivo é desenvolver a capacidade crítica, colaborativa e de construção de soluções a partir de diferentes contextos.

A estratégia de aprendizagem baseada em problemas (Problem-Based Learning PBL) possibilita discutir temas, trabalhar de forma cooperativa e desenvolver habilidades para resolução de problemas, bem como promover o pensamento de alto nível; como demonstrado por Silitonga et al. (2020) cujo estudo sobre habilidades de ordem superior de pensamento, em inglês "Higher order thinking skills" (HOTS) demonstrou que o HOTS do aluno de Física que estudou pela estratégia de resolução de problemas é maior do que o dos alunos pela estratégia de ensaio.

A PBL usualmente envolve aprendizagem colaborativa e pode usar recursos tecnológicos como elemento facilitador e catalisador. Busca motivar o diálogo entre os alunos, a realização de pesquisas, 
promovendo a autonomia dos mesmos (SOUZA e FONSECA, 2017). Neste aspecto, considera-se uma metodologia que contribui para a aprendizagem da Física.

\subsection{O Ensino de Física}

A Física é a ciência que estuda os fenômenos da natureza, leva-nos ao conhecimento dos fenômenos naturais, no cotidiano, na Terra, em outras galáxias; enfim, em todo o universo. Na classificação da Coordenação de Aperfeiçoamento de Pessoal de Nível Superior CAPES $^{9}$ a Física é uma área de conhecimento das Ciências Exatas e da Terra. Além disso, é componente curricular do Ensino Médio, iniciando no primeiro ano, tem seus conteúdos e currículo embasados pelos Parâmetros Curriculares Nacionais (PCNs, 2004) e Base Nacional Comum Curricular (BNCC, 2018). Divide-se em Física Experimental, com investigação de propriedades e transformações em ambiente laboratorial, e teórica com sistematização dos resultados de experimentos, estabelecendo relações entre conceitos e grandezas.

Segundo os PCNs (2004) o conhecimento da Física torna-se indispensável à formação da cidadania contemporânea desde que incorporado à cultura e integrado como instrumento tecnológico. $\mathrm{O}$ documento apregoa que o ensino de Física, no ensino médio: "contribua para a formação de uma cultura científica efetiva, que permita ao indivíduo a interpretação dos fatos, fenômenos e processos naturais, situando e dimensionando a interação do ser humano com a natureza como parte da própria natureza em transformação" (PCN, 2004, p.8).

Decorridos quase duas décadas dos PCNs, com as novas formulações da Base Nacional Comum Curricular (BNCC, 2018) o currículo do ensino médio foi organizado por meio da oferta de diferentes arranjos curriculares, em consonância com o contexto local e a possibilidade dos sistemas de ensino. As disciplinas como Química, Física e Biologia compõem o itinerário formativo de Ciências da Natureza e suas Tecnologias.

De acordo com Moreira (2018) houve um período de valorização do ensino da Física. Corroborando com Moreira, Rosa (2012) menciona que o entusiasmo com desenvolvimento da ciência e da tecnologia teve início na década de 60, o que levou à valorização do ensino de Ciências, em particular à Física.

Entretanto, como os autores indicam, esse ensino está em crise. Questões como redução da carga horária semanal de 6 horas-aula para menos de 2 horas por semana e escassez de laboratório de ciências dificultam a aprendizagem. Destaca a exígua participação de professores do ensino básico em publicações. Além disso, ressalta que muitas pesquisas que poderiam contribuir para o ensino, acabam por não modificá-lo na prática. Continua-se preparando os alunos para aprovarem em provas e vestibulares, sem aprenderem o que Ihes foi ensinado (MOREIRA, 2018; ROSA, 2012).

Outro fator destacado é a ausência das Tecnologias da Informação e Comunicação (TIC) no ensino da física e de outras disciplinas. Segundo Moreira (2018, p.76):

\footnotetext{
${ }^{9}$ A Física segundo a CAPES: - Física Geral; - Áreas Clássicas de Fenomenologia e suas Aplicações; - Física das Partículas Elementares e Campos; - Física Nuclear; - Física Atômica e Molecular; - Física dos Fluídos, Física de Plasmas e Descargas Elétricas; - Física da Matéria Condensada. Disponível em: <http://www.capes.gov.br/avaliacao/tabela-de-areas-de-conhecimento>.

<http://fisio.ic.usp.br:4882/posgraduacao/bolsas/capesproex_bolsas/tabela_areas.html>
} 
"Simulações computacionais, modelagem computacional, laboratórios virtuais deveriam estar naturalmente integrados ao ensino de Física no século XXI. Celulares também poderiam fazer parte dessa tecnologia que deveria permear o ensino de Física nos dias de hoje".

Corroborando com Moreira a proposta da BNCC (2018), enfatiza o compromisso de explicitar os fundamentos científico-tecnológicos da produção dos saberes e promover a articulação entre diferentes áreas do conhecimento. Na próxima seção aborda-se o agente conversacional, uma tecnologia que está ganhando espaço na educação e suas contribuições no ensino da Física.

\subsection{Agente conversacional e a Física}

O agente conversacional também designado (chatbot, chatterbot ou assistente virtual) caracteriza-se por uma entidade que simula uma conversação entre o homem e a máquina (TAROUCO, 2018; ARAVIND, 2018; GRAESSER, 2018). Os chatterbots são ferramentas tecnológicas que permitem simular conversas com seres humanos em linguagem habitual. Os estudos para construir sistemas de conversação iniciaram-se na década de 60, seguiram nas décadas seguintes e persistem nos dias atuais (GRAESSER, 2004, 2018). Entre outras funções, na educação, são utilizados para instruir o estudante durante o aprendizado de conteúdos específicos (PASCHOAL, MOZZAQUATRO, FALKEMBACH, 2017).

Tarouco et al. (2018) investigou a intervenção do agente conversacional/entidade virtual em sua capacidade de estimular alunos a refletirem, a partir das interações com o agente, desta forma ampliar a ZDP. Enfatiza que para ocorrer ampliação da ZDP o agente deve ser programado para criar eventos que promovam o estímulo ao pensamento por meio de perguntas instigantes.

Testou o potencial das entidades virtuais para o desenvolvimento da aprendizagem colaborativa e a extensão da ZDP com a tutora METIS (Mediadora de Educação em Tecnologia Informática e Socializadora). A partir do histórico (logs) do sistema da METIS, os registros dos alunos de EAD (Educação a Distância) com esta entidade foram extraídos e transcritos. Os dados também comprovaram que a entidade virtual pode atuar no campo emocional, estimulando e motivando o aluno, reduzindo o sentimento de isolamento e falta de integração relatada pelos alunos em cursos de educação à distância (TAROUCO et al. 2020).

A evolução na criação dos chatterbots chegou até o modelo da A.L.I.C.E ${ }^{10}$, baseado no padrão Artificial Intelligence Markup Language (AIML) ${ }^{11}$, para representação do conhecimento. A programação, assim como nos primeiros chatterbots quanto nos atuais, são baseados em AIML, a base de conhecimento é criada e mantida pelo gestor do chatterbot (Botmaster), uma pessoa que, manualmente, insere 0 conhecimento na base (TAROUCO et al. 2018; DANTAS 2019; LEONHARDT et al. 2003).

Para que o aplicativo simule o diálogo por meio de uma interação na linguagem natural, em forma de texto ou voz, com um humano, é necessário à entrada (humana) e a resposta (agente). A linguagem de programação permite configurar e programar as respostas do agente conversacional,

${ }^{10}$ O A.L.I.C.E (Artificial Linguistic Internet Computer Entity) é um chatterbot criado na Lehigh University por Richard S. Wallace, ativada em 1995, sendo um dos robôs mais populares da atualidade (LEONHARDT et al. 2003).

11 O AIML foi desenvolvido pela comunidade de software livre Alicebot e pelo Dr. Richard S. Wallace durante 1995-2000. Disponível em:<https://www.tutorialspoint.com/aiml/aiml_introduction.htm> 
usando recursos avançados, mesmo sem noções de programação. Neste modelo, o conhecimento é representado na forma (<pattern $><$ template $>$ ), o gestor do chatterbot tem que pensar nas interações que o chatterbot vai ser capaz de responder, e desta maneira inserir nas tags (TAROUCO et al. 2018; LEONHARDT et al. 2003). Nesta perspectiva, a partir de uma boa base de programação, o agente consegue manter uma conversa com o aluno, através de perguntas realizadas pelo estudante, representando um tutor pedagógico que provê suporte ao ensino de conteúdos (HERPICH et al.2016, GRAESSER et al., 2018).

No cenário de desenvolvimento de bots, o Projeto AVATAR ${ }^{12}$ (Ambiente Virtual de Aprendizagem e Trabalho Acadêmico Remoto) desenvolvido pela Universidade Federal do Rio Grande do Sul (UFRGS, 2019), vem pesquisando desde 2016, formas para facilitar a aprendizagem através de laboratórios virtuais e mundos virtuais com o uso da plataforma Open Simulator; além da implementação de agentes conversacionais agregados a estes laboratórios.

No estudo de Herpich et al. (2017) sobre mundos virtuais encontram-se duas classes de agentes conversacionais que foram projetados e implementados: (1) agentes voltados para apresentar recursos laboratoriais e esclarecer dúvidas sobre os recursos ou sobre conhecimentos pré-existentes, relativos à compreensão do experimento; (2) agentes que fornecem orientação na utilização de experimentos e apresentam questões que promovem a reflexão sobre o experimento e seus resultados.

Para validar seu projeto, aplicou-o com 32 estudantes de graduação de diferentes áreas, os quais realizaram um experimento com circuitos elétricos e os princípios de aprendizagem de eletricidade por meio de experiências em mundos virtuais. O trabalho expôs o princípio de que uma pessoa aprenderia por meio da descoberta e da experiência embasada na aprendizagem experiencial do Ciclo de Kolb. Em seus resultados apontam o uso potencial do agente conversacional em aumentar as interações no mundo virtual (HERPICH et al. (2017).

Prosseguindo às pesquisas desenvolvidas no âmbito do projeto AVATAR, contemplam-se os trabalhos que também implementaram um agente no mundo virtual, como no caso de Tibola (2018, p. 95): "desenvolveu-se um ambiente tridimensional ao qual o avatar do aluno é posicionado na entrada do mundo virtual. Neste local, o aluno tem o primeiro contato com um agente virtual (chatterbot), chamado de Tutora ATENA". A ATENA é um agente conversacional incorporado implementado via NPC (non-player character) e tem capacidade de dialogar e acompanhar o aluno durante seu tempo no cenário virtual. Este agente foi preparado para atuar no campo conceitual de Física no ensino médio (TAROUCO, 2018; TIBOLA, 2018).

Aravind e colaboradores (2018) reforça que o ensino de Física exige uma investigação autêntica, pautada no mundo real, com desenvolvimento da alfabetização científica. Desenvolveu um tutor baseado em computador para os alunos aprenderem os conteúdos de energia e potência. A metodologia envolveu inicialmente a apresentação de uma aula em vídeo curto (cerca de 5 minutos), após os alunos acessam o software intitulado: "computer-based tutor" que fora programado pelo professor, que instiga com perguntas sobre os conceitos aprendidos na aula, o tutor fornece suporte

12 O Projeto AVATAR é voltado para o ensino de ciências, área que tem sérias lacunas em termos de laboratórios para o desenvolvimento de experiências dos alunos. (TAROUCO e KRASSMANN,2018).

Disponível em: http://penta3.ufrgs.br/AVATAR/Livro/AVATAR-Cogni\%C3\%A7\%C3\%A3o-AprendizagemMundoVirtual-2018.pdf 
para preencher lacunas em seus conhecimentos. A implantação desse tutor para uso dos alunos foi possível por meio do TutorShop, um sistema de gerenciamento de alunos feito para ser usado com tutores inteligentes hospedado pela Carnegie Mellon University.Todos os recursos usados para este exercício estão disponíveis gratuitamente para fins educacionais, de modo que professores e alunos de várias origens econômicas podem fazer uso desses recursos.

A metodologia ativa contemplada na pesquisa de Aravind et al. (2018) foi a estratégia de resolução de problemas (Based Problem Learning) PBL. Ao tentar resolver problemas, os alunos são solicitados a fazer uma conexão entre os conceitos aprendidos em uma aula de teoria, e o problema em questão. O tutor projetado teve como objetivo fornecer treinamento intenso de problemas para os alunos. Para tanto, usou problemas simples, diferente daqueles que fariam num teste típico.

Dantas e colaboradores (2019) desenvolveu um chatbot com tecnologias de processamento de linguagem natural e inteligência artificial para atuar no ensino e aprendizagem do componente curricular da disciplina de Física, denominou-o Astrobot, disponibilizando o chatbot nos aplicativos dos alunos tais como: WhatsApp, Facebook Messenger e Telegram. Utilizou a linguagem de programação Python ${ }^{13}$ para criar a ferramenta. Primeiramente, construiu uma ferramenta para os docentes inserirem os conteúdos didáticos, permitindo "treinar" o bot com esses conhecimentos específicos.

Avaliou o aplicativo com quatro turmas do primeiro ano do ensino médio. Os alunos foram orientados a utilizar a aplicação durante a realização de exercícios, após as aulas no período de quatro semanas. O chatbot ajudou o docente a obter um feedback dos alunos em relação ao conteúdo proposto em sala de aula. Os conteúdos abordados pelo chatbot foram: velocidade, cinemática, vetores, movimento uniformemente variado, trajetória.

Graesser et al. (2004) desenvolveu o primeiro AutoTutor com a linguagem de programação Java, hospedado em um servidor denominado "Pentium-based". A versão mais recente, daquela época, foi desenvolvida em arquitetura modular; no entanto, não a apresentou. O software hospedado no servidor tem um conjunto de bancos de dados permanentes, incluindo cinco classificações (repositório de scripts de currículo, módulos de linguística computacional, corpus de documentos, glossário, espaço de Análise de Semântica Latente-LSA).

O método de aprendizagem proposto pelo AutoTutor visava a apresentação de questões ou problemas que requerem, aproximadamente, um parágrafo de informações para responder. A pergunta utilizada como exemplo no campo conceitual da Física:

\footnotetext{
"Suponha que um menino está em um elevador em queda livre e segura as chaves bem na frente de seu rosto e depois a solta. O que irá acontecer com as chaves? Explique o porquê. "Outro exemplo pergunta é "Quando um carro sem encostos de cabeça nos bancos é atingido por trás, os passageiros frequentemente sofrem lesões no pescoço (GRAESSER et al, 2004, p. 183).
}

Graesser et al. (2018) continuou pesquisando a aprendizagem mediada pelos tutores virtuais. Abordaram, brevemente alguns dos ambientes de aprendizagem típicos, focados no ensino da Física, incluindo seus benefícios e potenciais habilidades; tais como: leitura de livro didáticos, professores/palestras, ambientes de aprendizagem e descoberta, tutoria humana, animação,

\footnotetext{
${ }^{13}$ Python é uma linguagem de programação de alto nível, interpretada, de script, imperativa, orientada a objetos, funcional, de tipagem dinâmica e forte (DANTAS, et al, 2019).
} 
multimídia, simulação interativa, sistemas inteligentes de tutoria, jogos, agentes de conversação animados.

Nas análises de Graesser et al (2018) comparou-se os métodos pedagógicos para mediação dos conteúdos da disciplina Física, chegando às seguintes considerações: os ganhos de aprendizagem com o AutoTutor (agente conversacional) são superiores à leitura de um livro sobre os mesmos conteúdos por um período de tempo equivalente. As comparações foram feitas entre AutoTutor e tutores humanos por meio de comunicação mediada por computador. Os ganhos na aprendizagem foram equivalentes para alunos com um grau moderado de conhecimento em Física, mas tutores humanos especializados prevalecem quando os alunos especificam as dúvidas e dialogam. (GRAESSER et al. 2018). Além disso, destaca as possibilidades de interação social, geradas pelo tutor virtual.

De acordo com os estudos, encontram-se três razões porque os agentes contribuem para a aprendizagem. A primeira é que a tutoria individual é um dos métodos mais eficazes em apoio ao aluno. A segunda é que os agentes gerados por computador podem aplicar tutoria de forma consistente e confiável. Finalmente, os agentes podem modelar; ou seja, demonstrar a maioria das atividades e abordagem de aprendizagem que envolva interações entre pessoas e entre pessoas e os recursos tecnológicos (GRAESSER et al. 2018).

ANWARULLO (2019) da Universidade de Computação da Indonésia desenvolveu o aplicativo "Einstein Chatbot" para ajudar os alunos com uma compreensão suplementar do ensino na escola, para ser usado em casa. Utilizou API ${ }^{14}$ do Google com base no Android e a tecnologia Dialogflow ${ }^{15}$ é um software pago disponibilizado pelo Google que permite a programação em linguagem natural do bot. A realidade descrita pelo autor é que nas escolas secundárias, o ensino profissionalizante requer 0 conhecimento de Física. Os conteúdos abordados pelo chatbot envolvem os conhecimentos de grandezas (energia, massa, tempo). Inicialmente havia uma abordagem do professor, uma explicação do conteúdo; além disso, o "Einstein Chatbot" era carregado com os conteúdos para realizarem atividades, tirarem dúvidas.

Leonhardt et al. (2003) desenvolveu o chatbot Elektra a partir da base de programação da A.L.I.C.E, cujo objetivo inicial foi interagir com alunos do ensino secundário para fins de apoiar a aprendizagem em Física. Posteriormente, foi utilizada com alunos do Curso de Especialização a Distância em Informática na Educação na disciplina Internet para Educadores, acrescentando em sua base de conhecimento dados e conceitos sobre Redes de Computadores e Internet.

À medida que era implementada, outras funcionalidades foram inseridas como: hiperlink dando acesso a conteúdos de pesquisa, e possibilidade de múltiplas respostas para cada categoria. Dentre os benefícios destacados com seu chatbot no ensino a distância, está a capacidade de sanar dúvidas dos alunos remotamente, realizando o papel de um tutor virtual, diminuindo a percepção de solidão levantada pelos alunos do ensino a distância. O artigo demonstrou os ajustes minuciosos na

\footnotetext{
${ }^{14}$ API é um conjunto de rotinas e padrões de programação para acesso a um aplicativo de software ou plataforma baseado na Web. A sigla API refere-se ao termo em inglês "Application Programming Interface" que significa em tradução para o português "Interface de Programação de Aplicativos".

15 O Dialogflow é um conjunto de desenvolvimento para criar interfaces de conversa para sites, aplicativos móveis, plataformas de mensagens, interfaces (como bot de chat) que permitem interações naturais e ricas entre seus usuários e suas empresas. Fonte: https://dialogflow.cloud.google.com/. Acesso: Out/2020.
} 
programação, em relação ao processamento da linguagem natural e uso da inteligência artificial, que contribuem fortemente para a implantação de novos agentes.

\section{CONSIDERAÇÕES FINAIS}

Os agentes conversacionais têm recebido maior interesse na atualidade, destacando-se como uma tecnologia que proporciona alta capacidade de interação, de forma dinâmica com possibilidade de fornecer feedback para o aluno. Este artigo apresentou uma revisão sistemática de literatura, cujo critério de inclusão contempla pesquisas aplicadas envolvendo o uso de chatbot na disciplina de Física no Ensino Médio. Pesquisou-se as bases de dados da Scopus e do Google Scholar com os seguintes descritores "chatbot" and "high school" and "physics", no período delimitado de 2016 a 2020 com retorno de 410 artigos, dos quais apenas oito fizeram parte desta pesquisa.

Verificou-se que as pesquisas sobre a inserção dos agentes conversacionais no campo conceitual da Física no Ensino Médio estão em ascensão, mas são insuficientes as publicações para este nível educacional, considerando-se um vasto campo a ser estudado. Contudo, cabe destacar que a escolha dos termos relacionados à Física e ao chatbot utilizados na pesquisa foi realizada a partir do conhecimento empírico na área, e este fato pode ser considerado uma das limitações deste estudo.

A partir da análise dos estudos, verificaram-se três motivos pelos quais os agentes contribuem para a aprendizagem. Pontuados pela tutoria individual que ele proporciona capacidade de fornecer tutoria consistente e confiável, capacidade de demonstração da atividade proposta.

As contribuições de um agente conversacional na disciplina de Física são contempladas pela: capacidade de modelar; ou seja, simular experimentos; ampliação da ZPD na medida em que o agente instiga a reflexão, aumento na motivação nas aulas de física. A abordagem de uma metodologia ativa de ensino, foi referendada nos artigos pesquisados, e apontam para inovações no ensino, em que a participação dos alunos é mais efetiva, tornando-se responsáveis por sua aprendizagem.

A síntese dos dados foi demonstrada descritivamente na seção envolvendo os agentes conversacionais e a Física. Procurou-se averiguar e descrever os conteúdos trabalhados, os fundamentos teóricos de aprendizagem.

A pesquisa de Aravind (2012) detalha extraordinariamente a metodologia utilizada, baseada na resolução de problemas. Nas pesquisas de Herpich et al (2017) a teoria subjacente está embasada na Aprendizagem Experiencial de Kolb. Os estudos de Tarouco e Silveira (2018) abordam a teoria Sócio Interacionista de Vygotsky relacionando-a positivamente com as interações com agente conversacional.

Observou-se nos estudos analisados a descrição minuciosa da linguagem de programação do chatbot e dos recursos usados que podem beneficiar futuras pesquisas neste quesito. Dentre as linguagens de programação tem-se a Artificial Intelligence Markup Language ou Linguagem de Marcação de Inteligência Artificial, Phyton e Java.

O estudo estrangeiro de ANWARULLO (2019) apresenta contribuições quanto a linguagem de programação, a metodologia empregada foi através da estratégia de resolução de problemas. Outro estudo desenvolvido por Graesser (2018) também utiliza PBL. Destaca-se que dos oito artigos, cinco utilizaram PBL. Desta forma podendo indicar uma metodologia eficaz no ensino da Física 
Postula-se que na prática da educação, as prerrogativas da BNCC (2018) e PCN (2004) tornem-se realidade, destacando a priorização e valorização dos conhecimentos científicos e a inserção da tecnologia. Neste aspecto, que os conhecimentos em Física incluam a compreensão do conjunto de equipamentos e procedimentos, técnicos ou tecnológicos, do cotidiano doméstico, social e profissional.

Em trabalhos futuros serão realizadas novas pesquisas envolvendo o uso do agente conversacional, que se reflita numa metodologia ativa de aprendizagem, tal como referendado nos trabalhos de Herpich (2017); Aravind (2018) e Tarouco et al. (2018).

\section{REFERÊNCIAS}

ARRUDA, Juliana Silva. CASTRO, Aires Filho. Tecnologias Digitais e a Aprendizagem: Perspectivas para Emergência de Zonas e Desenvolvimento Proximal. Renote, V. 16 № 2, dezembro, 2018. Disponível em: https://seer.ufrgs.br/renote/article/viewFile/89265/51505. Acesso: Nov./2020.

ARAVIND, V. R., \& MCCONNELL, M. K. (2018). A computer-based tutor for learning energy and power.10 (3), 174-185. doi:10.18844/wjet.v10i3.3558

ANWARULLOH, T. P. AGUSTIA, R. D. Development Of The Chatbot Einstein Application As A Virtual Teacher Of Physical Learning In The House Using Android Based Google Dialogflow Api. Universitas Komputer Indonesia. 2019.

https://elibrary.unikom.ac.id/id/eprint/900/14/22.10114509_TB\%20PRASSETYO\%20A_JURNAL\%20 DALAM\%20BAHASA\%20INGGRIS.pdf

Cognição e aprendizagem em mundo virtual imersivo [recurso eletrônico] / organizadores Liane Margarida Rockenbach Tarouco, Patrícia Fernanda da Silva [e] Fabrício Herpich ; coordenado pela SEAD/UFRGS. - 2. ed. - Porto Alegre: Editora da UFRGS, 2020. 355 p.: pdf.

\section{Disponível}

em:<https://www.lume.ufrgs.br/bitstream/handle/10183/210290/001115215.pdf?sequence=1\&isAll owed $=\mathrm{y}>$

DANTAS, A. C. TORRES, H. FARIA, I. P. ARAÚJO W. GUIMARÃES, G. MACHADO G. ALMEIDA, A. V. NASCIMENTO, M. Z. AstroBot: Um chatbot com inteligência artificial para auxiliar no processo de ensino e aprendizagem de Física. (2019). VIII Congresso Brasileiro de Informática na Educação.1196. 10.5753/CBIE.WCBIE.2019.1196.

GRAESSER, A.C., Lu, S. JACKSON, G.T. et al. AutoTutor: A tutor with dialogue in natural language. Behavior Research Methods, Instruments, \& Computers 36, 180-192 (2004). https://doi.org/10.3758/BF03195563

GRAESSER, A. C., FRANCESCHETTI, D., GHOLSON, B., \& CRAIG, S. Learning newtonian physics with conversational agents and interactive simulations. Developmental Cognitive Science Goes to School. (pp. 157-172). 2018. Taylor and Francis.

https://doi.org/10.4324/9780203837535

HECK, Carine, COELHO, Karine S., SIMÃO, José P. S., SILVA, Isabela N., SILVA, Juarez B., BILESSIMO, Simone M. S. Experiência de Integração da Experimentação Remota no Ensino 
de Física do Ensino Médio: Percepção dos Alunos. Revista Novas Tecnologias na Educação (RENOTE), 14 (2), p. 1-10, 2016.

HERPICH, F.; VOSS, G. B.; NUNES, F. B.; MEDINA, R. D. Three-Dimensional Virtual Environment and NPC: A Perspective about Intelligent Agents Ubiquitous. In: Francisco Milton Mendes Neto; Rafael de Souza; Alex Sandro Gomes. (Org.). Handbook of Research on 3-D Virtual Environments and Hypermedia for Ubiquitous Learning. 1ed.: IGI Global, 2016, v. 21, p. 510-536. DOI: 10.4018/978-1- 5225-0125-1.ch021.

HERPICH F., Filho T.A.R., TIBOLA L.R., FERREIRA V.A., TAROUCO L.M.R. (2017) Learning Principles of Electricity Through Experiencing in Virtual Worlds. In: Beck D. et al. (eds) Immersive Learning Research Network. iLRN 2017. Communications in Computer and Information Science, vol. 725. Springer, Cham. https://doi.org/10.1007/978-3-319-60633-0_19

HERPICH, F. Recursos Educacionais em Realidade Aumentada para o Desenvolvimento Da Habilidade De Visualização Espacial em Física. Tese PPGIE/UFRGS. Orientadora: Liane Margarida Rockenbach Tarouco Porto Alegre-RS. 2019. Disponível em:

<https://lume.ufrgs.br/handle/10183/199101>. Acesso: Julho/2020.

KITCHENHAM, B., BRERETON, O. P., BUDGEN, D., TURNER, M., BAILEY, J., and Linkman, S. (2009). Systematic literature reviews in software engineering-a systematic literature review. Information and software technology, 51(1):7-15

KLOCK, A. C. T. Mapeamento e Revisões Sistemáticos da Literatura: um guia prático e teórico. Cadernos de Informática. Volume 10. № 1. 2018. Fonte:

https://www.seer.ufrgs.br/cadernosdeinformatica/article/view/v10n1201801-09/49901 Acesso em: out/2020

KRASSMANN, Aliane Loureiro Krassmann. TAROUCO, Liane Margarida Rockenbach. Chatbots na educação: uma Revisão Sistemática da Literatura. Renote, V. 16. №1, dezembro, 2018.

LEONHARDT, Michelle Denise. CASTRO, Daiane Dorneles De. DUTRA, Renato Luís De Souza. TAROUCO, Liane Margarida Rockenbach ELEKTRA: Um Chatterbot para Uso em Ambiente Educacional. RENOTE, v. 1, n. 2 (2003).

Disponível em:<http://penta3.ufrgs.br/ elektra/info/artigos/chatterbot-Elektra\%5B1\%5D.PDF>

MOREIRA, M. A.Uma análise crítica do ensino de Física. Estudos Avançados 32 (94), 2018.

MOREIRA, M. A. Mapas conceituais e aprendizagem significativa. Instituto de Física. UFRGS. 2012. Disponível em: <https://www.if.ufrgs.br/ moreira/mapasport.pdf>. Acesso em 12 ago. 2020.

MOTA, A.; WERNER DA ROSA, C. Ensaio sobre metodologias ativas: reflexões e propostas. Revista Espaço Pedagógico, v. 25, n. 2, p. 261-276, 28 maio de 2018.

PASCHOAL, Leo Natan. CHICON, Patrícia M. FALKEMBACH, Gilse Antoninha M. Ubibot: Um Agente Conversacional Ciente do Contexto de Aprendizagem do Usuário. CINTED-UFRGS. V. 14 No 1, julho, 2016.

PASCHOAL, L. N. MOZZAQUATRO P.M.C. FALKEMBACH, G. A. M. Concepção, Implementação e Avaliação de um Agente Conversacional com Suporte à Aprendizagem Ubíqua. CINTED-UFRGS, V. 15 No 1, julho, 2017. 
ROSA. Cleci Werner. ROSA, Álvaro Becker. O ensino de ciências (Física) no Brasil: da história às novas orientações educacionais. Revista Iberoamericana de Educación n. ${ }^{0}$ 58/2 15/02/12. ISSN: $1681-5653$

SIlitongA, H. T. M., PANJAITAN, M., \& SUPRIYATI, Y. (2020). Problem solving based physics learning strategy to enhance students' higher order thinking skills. Paper presented at the Journal of Physics: Conference Series, 1567(4) doi:10.1088/1742$6596 / 1567 / 4 / 042104$

TAROUCO, Liane. SILVA, Fernanda. HERPICH, Fabrício. Cognição e aprendizagem em Mundo Virtual Imersivo / Organizadores: Liane Tarouco, Patrícia F. da Silva, Fabrício Herpich. Porto Alegre: Ed. UFRGS, 2019. 232 p. Disponível em:<http://penta3.ufrgs.br/AVATAR/Livro/AVATAR-Cogni\%C3\%A7\%C3\%A3o-AprendizagemMundoVirtual-2018.pdf $>$

TAROUCO, Liane. SILVEIRA, Clóvis. KRASSMANN, Aliane. Collaborative Learning with Virtual Entities. In: International Conference on Learning and Collaboration Technologies, 2018, Las Vegas, USA. 2018 HCI Human-Computer Interaction. New York USA: Springer, 2018. p. 480493.

TEIXEIRA, Odete P. Baierl. A Ciência, a Natureza da Ciência e o Ensino de Ciências. Ciênc. educ. (Bauru) vol.25 no.4 Bauru Oct./Dec. 2019 Epub Dec 20, 2019. https://doi.org/10.1590/1516731320190040001

TIBOLA, Leandro Rosniak. Fatores Ensejadores de Engajamento em Ambientes de Mundos Virtuais. Tese apresentada como requisito parcial para a obtenção do grau de Doutor em Informática na Educação UFRGS. Orientadora: Profa. Dra. Liane Margarida Rockenbach Tarouco. Porto Alegre: 2018.

VYGOTSKY, Liev Semionovich. A formação social da mente. São Paulo: Martins Fontes, 1989. . VYGOTSKY, Liev Semionovich. Mind in Society. Harvard University Press. 1978. Pensamento e Linguagem. 2. ed. São Paulo: Martins Fontes, 1998. 194p. 